\title{
Linking the gut microbiota to persistent symptoms in survivors of COVID-19 after discharge
}

\author{
Yaya Zhou ${ }^{\dagger}$, Jianchu Zhang ${ }^{\dagger}$, Dongmei Zhang, \\ Wan-Li Ma ${ }^{\star}$, and Xiaorong Wang \\ Department of Respiratory and Critical Care Medicine, Union Hospital, \\ Tongji Medical College, Huazhong University of Science and Technology, \\ Wuhan 430022, P. R. China \\ (Received Apr 16, 2021 / Revised Jun 22, 2021 / Accepted Jul 12, 2021)
}

Several follow-up studies have found that COVID-19 (coronavirus disease 2019) patients had persistent symptoms after discharge. Gut microbiota play an important role in human health and immune responses. Therefore, this study investigated the gut microbiota of recovered COVID-19 patients and the correlations between gut microbiota and persistent symptoms after discharge. Stool samples were collected from 15 recovered healthcare workers (HCWs) with COVID-19 at three months after discharge, in addition, stool samples were collected from 14 healthy controls (HCs) to perform $16 \mathrm{~S}$ rRNA gene sequencing between May and July 2020. Compared with HCs, recovered HCWs had reduced bacterial diversity at three months after discharge, with a significantly higher relative abundance of opportunistic pathogens, and a significantly lower relative abundance of beneficial bacteria. In addition, Escherichia unclassified was positively correlated with persistent symptoms at three months after discharge, including fatigue $(r=0.567, p=0.028)$, chest tightness after activity $(r=0.687, p=0.005)$, and myalgia $(r=0.523, p=0.045)$. Intestinibacter bartlettii was positively correlated with anorexia $(r=0.629, p=0.012)$ and fatigue $(r=0.545, p=0.036)$. However, Faecalibacterium prausnitzii was negatively correlated with chest tightness after activity $(r=-0.591, p=0.02)$, and Intestinimonas butyriciproducens was negatively correlated with cough $(r=-0.635, p=0.011)$. In conclusion, the gut microbiota of recovered HCWs with COVID-19 at three months after discharge was different from that of HCs, and altered gut microbiota was correlated with persistent symptoms after discharge, highlighting that gut microbiota may play an important role in the recovery of patients with COVID-19.

Keywords: COVID-19, recovered healthcare workers, gut microbiota, symptoms after discharge

\footnotetext{
${ }^{\dagger}$ These authors contributed equally to this work.

*For correspondence. (X. Wang) E-mail: rong-100@163.com / (W.L. Ma) E-mail: whmawl@aliyun.com; Tel.: +86-27-85726707; Fax: +86-27-85726 707

${ }^{8}$ Supplemental material for this article may be found at

http://www.springerlink.com/content/120956.

Copyright (C) 2021, The Microbiological Society of Korea
}

\section{Introduction}

COVID-19 (coronavirus disease 2019) is an infectious disease caused by severe acute respiratory syndrome coronavirus 2 (SARS-CoV-2), which has rapidly spread worldwide (Chan et al., 2020). Our previous studies reported data on the incubation period, clinical manifestations, virus shedding time, and complete genomic sequences of SARS-CoV-2 from healthcare workers (HCWs) with COVID-19 in the acute phase (Wang et al., 2020a, 2020b). Subsequently, we found that some of these healthcare workers (HCWs) had persistent symptoms, abnormal lung function, and abnormal lung images at three months after discharge (Liang et al., 2020); however, the mechanism of persistent abnormality in recovered HCWs is unclear.

The gut microbiota has a profound impact on human health and disease (Ichinohe et al., 2011; Belkaid and Hand, 2014; Schirmer et al., 2016), and several recent studies have suggested that the gut microbiota is altered in acute COVID-19 patients (Gu et al., 2020; Zuo et al., 2020a, 2020b, 2021; Yeoh et al., 2021) and is associated with fecal levels of SARS-CoV-2 (Zuo et al., 2020b), disease severity (Zuo et al., 2020b; Yeoh et al., 2021), several cytokines, and inflammatory markers (Yeoh et al., 2021). However, the long-term gut microbiota of recovered patients with COVID-19 and its influence on recovery are not clear. This study compared the fecal microbiota of recovered HCWs at three months after discharge with healthy controls (HCs) to identify correlations between gut microbiota and persistent symptoms after discharge.

\section{Materials and Methods}

\section{Study design and enrolled participants}

A cohort of 29 subjects was recruited, including 15 recovered HCWs from the above 76 recovered patients (Liang et al., 2020) with COVID-19 who were discharged from the Wuhan Union Hospital and 14 HCs between May and July 2020. All recovered HCWs met uniform discharge criteria according to the Chinese clinical guidance for COVID-19 pneumonia diagnosis and treatment issued by the National Health Commission (China National Health Commission). All recovered HCWs who received antibiotics, probiotics, or both within 3 months before enrollment were excluded. RNA in the stool specimens of recovered HCWs in the acute and recovery phases were extracted and tested for SARS-CoV-2 by real-time RT-PCR using a previously described protocol (Huang et al., 2020). HCs were recruited from healthy HCWs without COVID-19 at the Wuhan Union Hospital. All HCs were free of malignancy or gastrointestinal disease, without 
any history of antibiotics or chemotherapeutic drugs for at least 3 months before sample collection. The study was approved by the Ethics Committee of the Wuhan Union Hospital (2020-0149-02). All participants provided written informed consent prior to participation.

\section{Sample collection}

Twenty-nine stool samples from 15 recovered HCWs with COVID-19 and 14 HCs were collected (one stool sample per patient). Fresh stools from recovered HCWs with COVID-19 were collected during following-up, and fresh stools from HCs were collected during the recruitment process. Stool samples were processed in the laboratory within $30 \mathrm{~min}$ after collection and stored at $-80^{\circ} \mathrm{C}$ until analysis.

\section{DNA extraction and 16S rRNA gene sequencing}

The microbiome DNA was extracted using the MagPure Stool DNA KF kit B (Magen) following the manufacturer's instructions. DNA was quantified with a Qubit Fluorometer using the Qubit ${ }^{\circledR}$ dsDNA BR Assay kit (Invitrogen), and the quality was checked by running an aliquot on $1 \%$ agarose gel. Variable regions V3-V4 of the bacterial 16S rRNA gene were amplified using degenerate PCR primers $341 \mathrm{~F}$ (5'-ACTCC TACGGGAGGCAGCAG-3') and 806R (5'-GGACTACHV GGGTWTCTAAT- $\left.3^{\prime}\right)$. Forward and reverse primers were tagged with Illumina adapter, pad, and linker sequences. PCR enrichment was performed in a $50 \mu \mathrm{l}$ reaction containing $30 \mathrm{ng}$ template, fusion PCR primer, and PCR master mix. PCR cycling conditions were as follows: $94^{\circ} \mathrm{C}$ for $3 \mathrm{~min} ; 30$ cycles of $94^{\circ} \mathrm{C}$ for $30 \mathrm{sec}, 56^{\circ} \mathrm{C}$ for $45 \mathrm{sec}, 72^{\circ} \mathrm{C}$ for $45 \mathrm{sec}$, and a final extension for $10 \mathrm{~min}$ at $72^{\circ} \mathrm{C}$ for $10 \mathrm{~min}$. The PCR products were purified using AmpureXP beads and eluted in elution buffer. Libraries were qualified using the Agilent 2100 Bioanalyzer (Agilent). The validated libraries were used for sequencing on the Illumina MiSeq service (BGI) following the standard pipelines of Illumina, and two 300 bp pairedend reads were generated.

\section{Bioinformatic analysis of $16 \mathrm{~S}$ rRNA sequencing}

To obtain fragments of the target region, we used cutadapt v2.6 to cut the primer and adaptor contamination for reads that could match the primers. We set a window length of $30 \mathrm{bp}$, if the average quality value of the window was less than 20 , the end sequence of the read was cut from the beginning

Table 1. Demographic of recovered healthcare workers (HCWs) with COVID-19 and healthy controls (HCs)

\begin{tabular}{lccc}
\hline \multicolumn{1}{c}{ Demographic } & $\begin{array}{c}\text { Recovered HCWs } \\
(\mathrm{n}=15)\end{array}$ & HCs $(\mathrm{n}=14)$ & -value \\
\hline Age, years & $29.0(28.0-41.0)$ & $37.5(31.0-48.5)$ & 0.155 \\
Gender & $3(20.0)$ & $5(35.7)$ & 0.427 \\
$\quad$ Male & $12(80.0)$ & $9(64.3)$ & \\
$\quad$ Female & $22.7(20.9-24.8)$ & $24.1(21.0-26.3)$ & 0.432 \\
BMI, kg/m ${ }^{2}$ & & & \\
Comorbidities & $2(13.3)$ & $0(0.0)$ & 0.483 \\
\hline
\end{tabular}

BMI, body mass index

Data are $n(\%)$ or median (IQR). P values were calculated by Fisher's exact test or

Mann-Whitney $\mathrm{U}$ test. $p$-value $<0.05$ is considered to be statistically significant. of the window, and removed the reads whose final read length was less than $75 \%$ of the original read length. To obtain the final clean data, we also removed reads containing " $N$ " and low-complexity reads (10 consecutive ATCGs). FLASH (Fast Length Adjustment of Short reads, v1.2.11) was used for sequence splicing. The paired reads obtained by paired-end sequencing were assembled into a sequence by using the overlap relationship to obtain the tags of the hypervariable region. The splicing conditions were as follows: (1) the minimum matching length was $15 \mathrm{bp}$, and (2) the allowable mismatch rate in the overlapping area was 0.1 . The DADA2 (divisive amplicon denoising algorithm) method in QIIME2 was used to denoise and obtain Amplicon Sequence Variants (ASVs). The ASV is a $100 \%$ similar sequence. Thereafter, ASV representative sequences were taxonomically classified using the Ribosomal Database Project (RDP) Classifier v.2.2, with a minimum confidence threshold of 0.6 . Alpha diversity and beta diversity were estimated using MOTHUR (v1.31.2) and QIIME (v1.8.0). Significantly different species were determined using R (v3.4.1) based on the Wilcoxon test.

\section{Statistical analysis}

Median values with interquartile range (IQR) or mean \pm standard deviation (SD) were used to describe continuous variables, and absolute or relative frequencies were used to de-

Table 2. Symptoms, lung function, and lung images of 15 recovered HCWs with COVID-19 at three months after discharge

\begin{tabular}{lc}
\multicolumn{1}{c}{ Characteristics } & $\begin{array}{c}\text { Recovered HCWs } \\
(\mathrm{n}=15)\end{array}$ \\
\hline Symptoms & $12(80.0)$ \\
Any one of the following symptoms & $8(53.3)$ \\
Cough & $3(20.0)$ \\
Sputum & $5(33.3)$ \\
Chest tightness after activity & $3(20.0)$ \\
Anorexia & $3(20.0)$ \\
Diarrhea & $6(40.0)$ \\
Fatigue & $4(26.7)$ \\
Myalgia & \\
Lung function & $109.8 \pm 14.8$ \\
FVC (pred \%) & $101.1 \pm 11.1$ \\
FEV1 (pred \%) & $79.3 \pm 5.9$ \\
FEV1/FVC (\%) & $102.0 \pm 10.0$ \\
TLC (pred \%) & $108.4 \pm 20.9$ \\
RV (pred \%) & $105.1 \pm 15.0$ \\
FRC (pred \%) & $107.2 \pm 13.8$ \\
VC (pred \%) & $92.3 \pm 5.5$ \\
DLCO (pred \%) & $93.7 \pm 9.0$ \\
DLCO/VA (pred \%) & \\
Chest CT & $13(86.7)$ \\
Normal & $1(6.7)$ \\
Subpleural line & $2(13.3)$ \\
Interlobular septal thickening & $1(6.7)$ \\
GGO & \\
\hline FVC, forced vital capacity; FEV1, forced expiratory volume in one second; TLC, to- \\
tal lung capacity; RV, residual volume; FRC, functional residual capacity; VC, vital \\
capacity; DLCO, diffusion capacity for carbon; VA, alveolar volume; CT, computed \\
tomography; GGO, ground glass opacity. \\
Data are n (\%), or mean \pm SD. \\
\end{tabular}



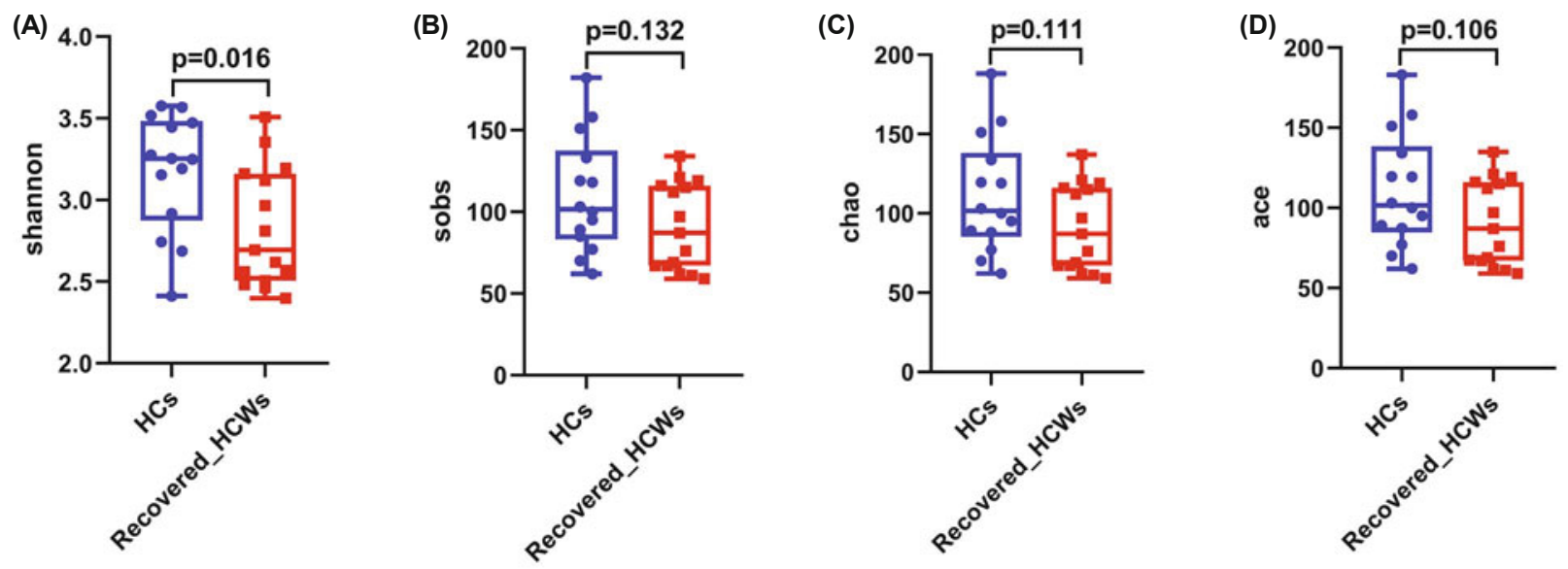

Fig. 1. Comparison of alpha diversity communities between recovered healthcare workers (HCWs) and healthy controls (HCs). (A) shannon, (B) sobs, (C) chao, and (D) ace. $p$-value $<0.05$ is considered to be statistically significant.

scribe categorical variables. We used the Mann-Whitney U test or independent t-test to analyze continuous variables and the $\chi 2$ test or Fisher's exact test for the analysis of categorical variables. For correlation analysis, Spearman's rank test was performed. Statistical analysis was performed using SPSS version 25.0 (IBM). A two-sided $p$-value of $<0.05$ was considered statistically significant.

\section{Results}

\section{Demographics and characteristics of the participants}

The demographic data of 15 recovered HCWs and 14 HCs are summarized in Table 1 . In addition, symptoms, lung function, and lung images of recovered HCWs at three months after discharge are summarized in Table 2 (Supplementary data Tables S1 and 2). The median age of recovered HCWs was 29.0 (28.0-41.0) years, with three (20\%) men and 12 (80\%) women. In comparison, the HCs comprised nine women and five men with a median age of $37.5(31.0-48.5)$ years. Two recovered HCWs had hypertension, and there were no comorbidities in the HCs. There was no difference between the two groups for age, sex, body mass index (BMI), and comorbidity. At least one symptom was reported by $80 \%$ of the recovered HCWs (12 of 15) at three months after discharge. The most common symptoms after discharge were cough $53.3 \%, 8$ of $15)$ and fatigue (40\%, 6 of 15), followed by chest tightness after activity (33.3\%, 5 of 15) and myalgia (26.7\%, 4 of 15). Although the mean values of all pulmonary function measurements were within normal limits in recovered HCWs (Table 2), recovered HCW 1 with abnormalities in total lung capacity (TLC), residual volume (RV), functional residual capacity (FRC), and recovered HCW 15 with abnormalities in forced expiratory volume in one second (FEV1)/forced vital capacity (FVC) were detected. Supplementary data Table S1 shows the pulmonary function data of the 15 recovered HCWs. Additionally, persistent lung computed tomography (CT) injuries at three months after discharge were seen in 2 recovered HCWs.

\section{Taxonomic differences of gut microbiota between recovered} HCWs and HCs

In total, 29 stool samples (15 stool samples from recovered HCWs and 14 stool samples from HCs) were sequenced, generating an average of 134,410 clean reads per sample (Supplementary data Table S3). Alpha diversity measured by four different methods were all lower in the recovered HCWs than in HCs, only the difference in shannon index reached statistical significance by Wilcoxon rank sum test (shannon, $\mathrm{p}=$ 0.016 ; sobs, $\mathrm{p}=0.132$; chao, $\mathrm{p}=0.111$; ace, $\mathrm{p}=0.106$ ) (Fig. 1 ). Principal coordinates analysis of Bray-Curtis distances indicated difference in the fecal microbiota between recovered HCWs and HCs $(\mathrm{P}=0.009)$ (Fig. 2). To investigate changes in the microbiota of recovered HCWs compared with HCs, we subsequently assessed relative abundance in the two groups at the phylum, family, genus, and species levels. At the phylum level, Firmicutes (mean 47.78\% vs. 62.34\%), Bacteroidetes (mean 38.27\% vs. 30.65\%), and Proteobacteria (mean 9.48\%

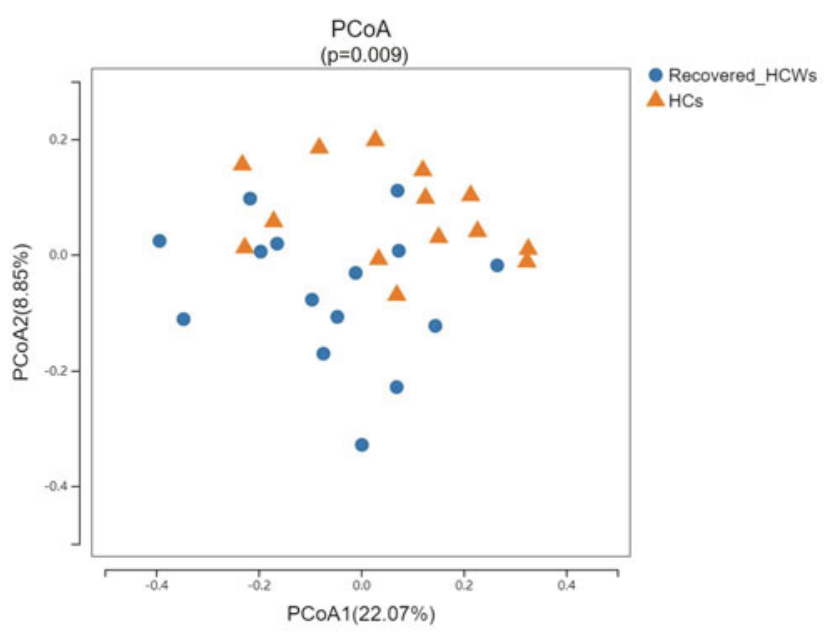

Fig. 2. The beta diversity results were assessed by principal coordinates analysis (PCoA) of Bray-Curtis distances between recovered HCWs and HCs. 
(A)

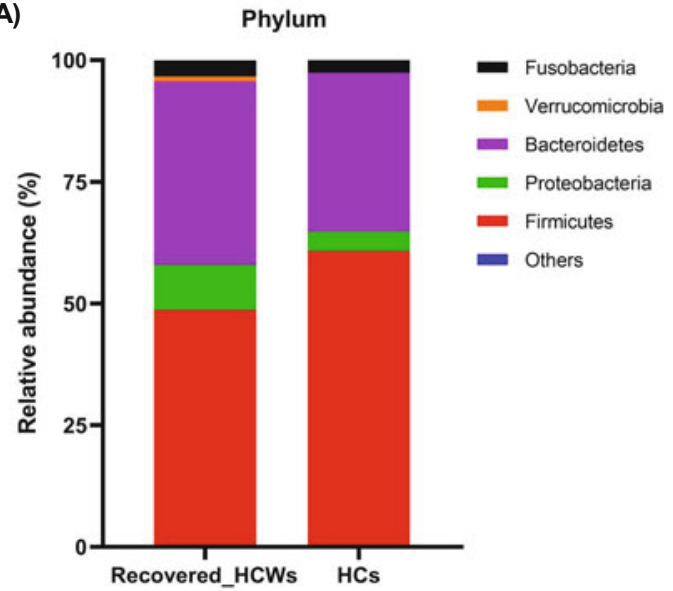

(B)

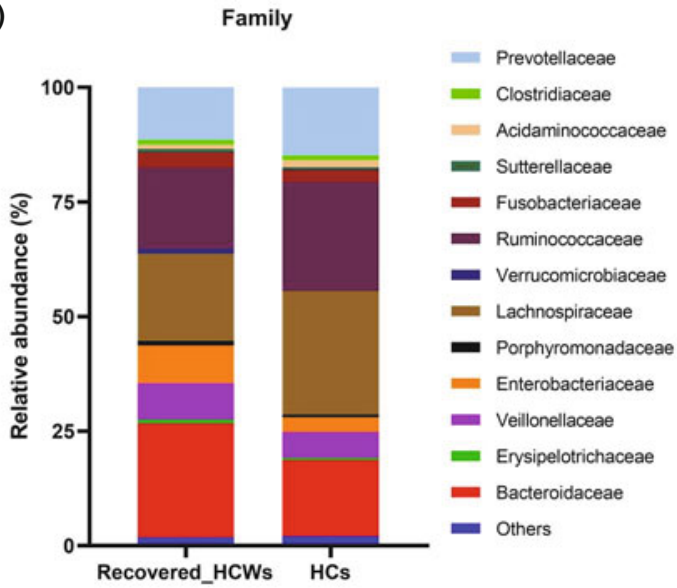

Fig. 3. Taxonomic distribution between recovered HCWs and HCs described by mean relative abundance. (A) Taxonomic distribution at the phylum level, (B) Taxonomic distribution at the family level.

vs. $4.13 \%)$ were the most dominant phyla in both recovered HCWs and HCs (Fig. 3A). The relative abundance of Actinobacteria was significantly increased in recovered HCWs with COVID-19 compared with HCs (mean $0.31 \%$ vs. $0.08 \%$, p $=0.035$, Mann-Whitney test) (Fig. 4A). At the family level, Lachnospiraceae and Desulfovibrionaceae were depleted in recovered HCWs (Figs. $3 \mathrm{~B}$ and $4 \mathrm{~B}$ ). At the genus level, Faecalibacterium, Roseburia, Fusicatenibacter, Ruminococcus,
Clostridium XVIII, Dorea, Butyricicoccus, Romboutsia, Intestinimonas, and Bilophila were depleted, and Escherichia, Flavonifractor, and Intestinibacter were enriched in recovered HCWs compared to HCs (Fig. 4C). At the species level, there were 20 bacterial taxa with a mean relative abundance $>10^{-5}$, showing distinct relative abundances between the two groups. Several opportunistic pathogens, including Escherichia unclassified, Intestinibacter bartlettii, Clostridium aldenense,

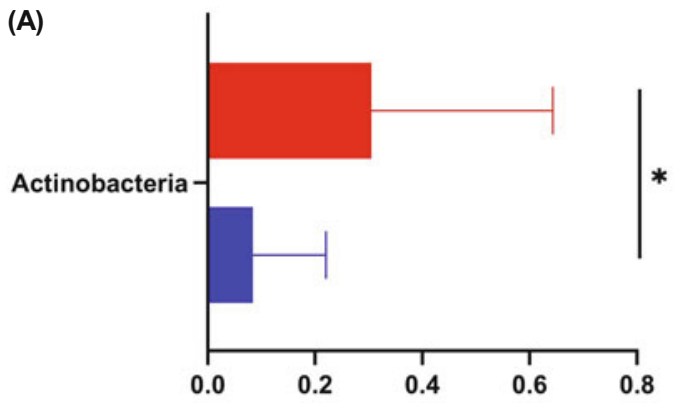

(B)

(C)

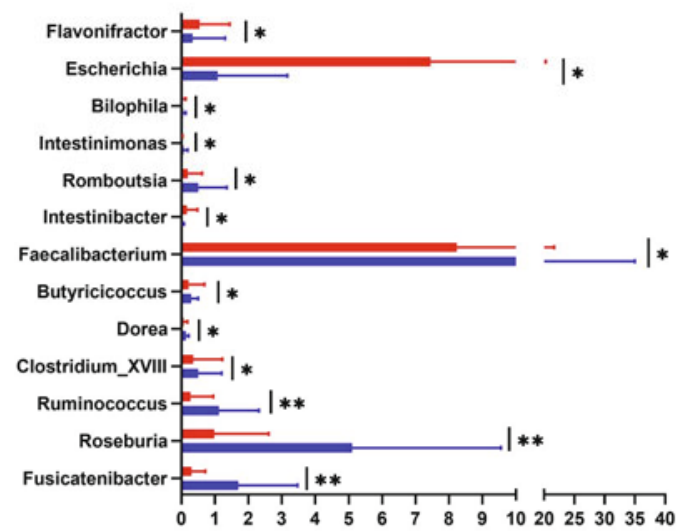

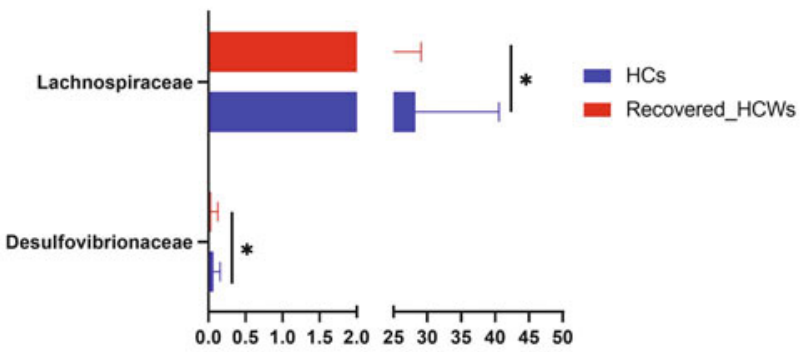

(D)

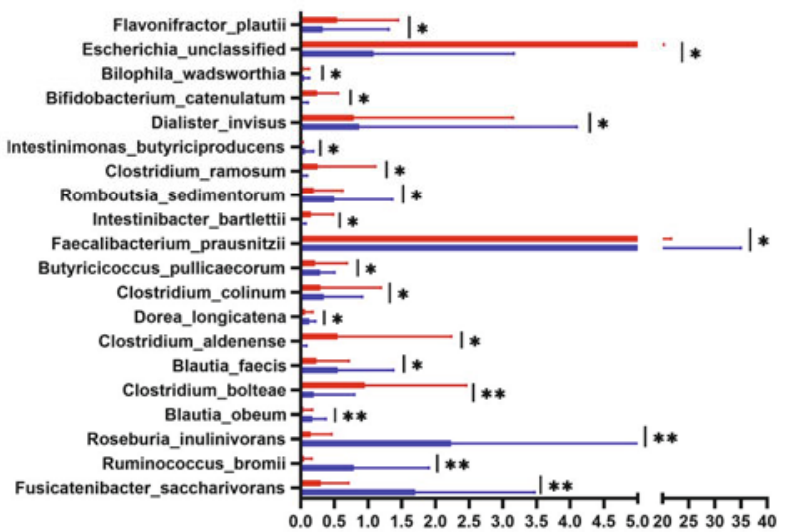

Fig. 4. Taxonomic differences of gut microbiota between recovered HCWs and HCs. Comparison of relative abundance at the bacterial phylum (A), family (B), genus (C), and species (D) between the two groups. $p$-value $<0.05$ is considered to be statistically significant, ${ }^{*} p<0.05,{ }^{* *} p<0.01$. 
inflammation-related pathogens including Clostridium bolteae, Flavonifractor plautii, and COVID-19 severity-related pathogen (Clostridium ramosum) were enriched in recovered HCWs. In addition, recovered HCWs demonstrated a further depletion of multiple bacterial species, including Faecalibacterium prausnitzii, Roseburia inulinivorans, Fusicatenibacter saccharivorans, Ruminococcus bromii, Blautia faecis, Butyricicoccus pullicaecorum, and Intestinimonas butyriciproducens (Fig. 4D). These results indicated that recovered HCWs with COVID-19 had significant alterations in fecal microbiota compared with HCs, characterized by enrichment of opportunistic pathogens and depletion of beneficial commensals (Supplementary data Table S4-7).

\section{Relationship between different species and symptoms after discharge}

Spearman's rank-based correlation test was performed to identify microbial species associated with persistent symptoms in recovered HCWs with COVID-19 at three months after discharge. We found that F. prausnitzii was negatively correlated with chest tightness after activity $(\mathrm{r}=-0.591, \mathrm{p}=$ 0.02 ), and I. butyriciproducens was negatively correlated with cough $(\mathrm{r}=-0.635, \mathrm{p}=0.011)$. Escherichia unclassified was positively correlated with fatigue $(\mathrm{r}=0.567, \mathrm{p}=0.028)$, chest tightness after activity $(\mathrm{r}=0.687, \mathrm{p}=0.005)$, and myalgia $(\mathrm{r}=$ $0.523, \mathrm{p}=0.045)$. Intestinibacter bartlettii was positively correlated with anorexia $(\mathrm{r}=0.629, \mathrm{p}=0.012)$ and fatigue $(\mathrm{r}=$ $0.545, \mathrm{p}=0.036$ ) (Fig. 5).

\section{Discussion}

As far as we known this was the first study to show the gut microbiota of recovered HCWs with COVID-19 at three months after discharge was different from that of HCs, and altered gut microbiota was correlated with persistent symptoms after discharge. These data suggest that depletion of salutary species may persist in most recovered patients despite SARS-CoV-2 virus clearance, suggesting exposure to SARS-CoV-2 infection may be associated with a more longlasting detrimental effect on the gut microbiota.

Several studies on the gut microbiota of COVID-19 patients have mainly focused on the acute phase of COVID-19. However, the composition of the gut microbiota at long

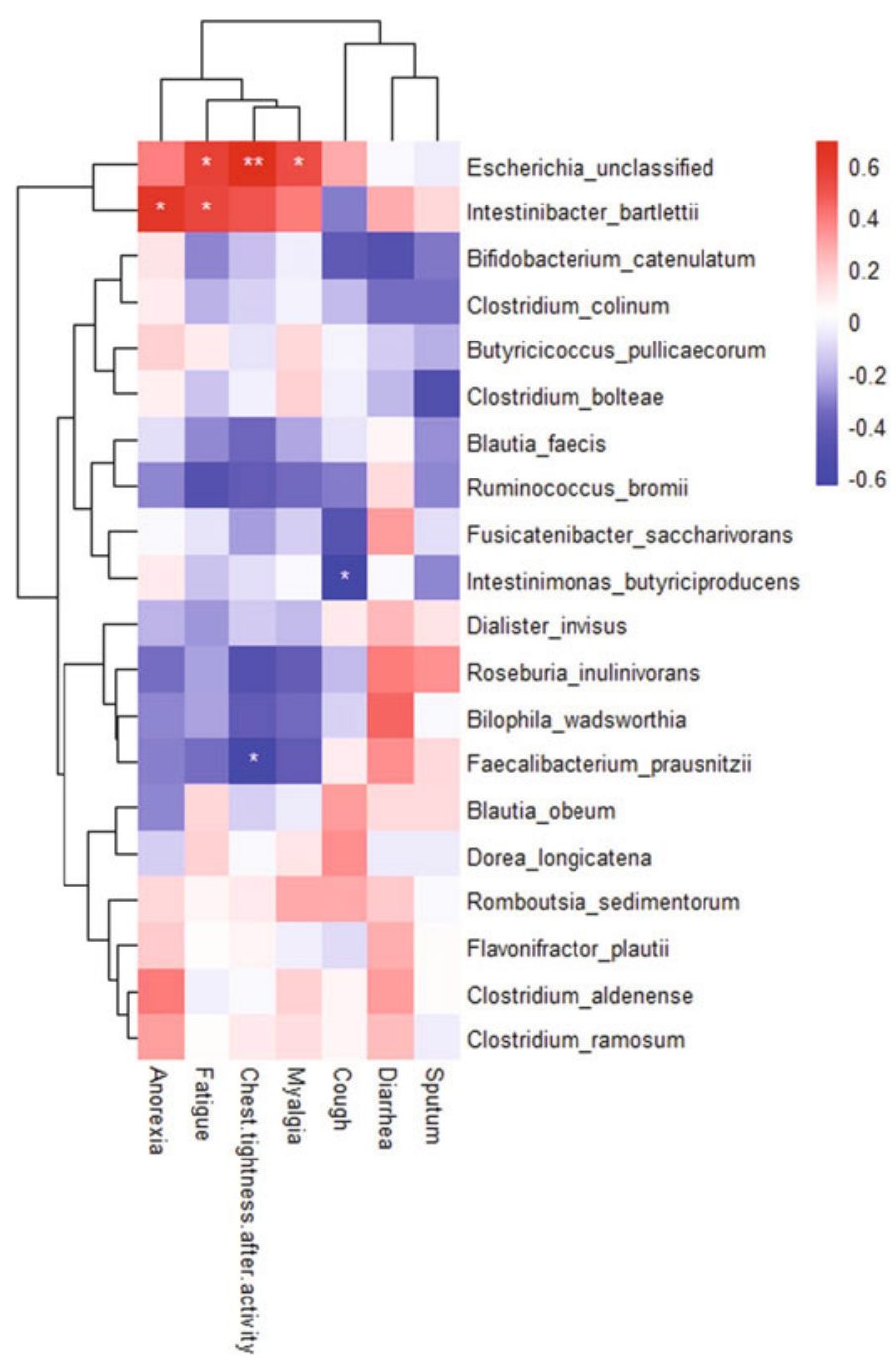

Fig. 5. Relationship between different species and persistent symptoms of recovered HCWs with COVID-19 at three months after discharge. Spearman's rank correlation coefficient is indicated using a color gradient: red indicates positive correlation; blue indicates negative correlation. $p$-value $<0.05$ is considered to be statistically significant, ${ }^{*} p<0.05,{ }^{* *} p<0.01$. 
term after discharge is not clear. Gu et al. (2020) have found that COVID-19 patients had a significantly higher relative abundance of Streptococcus, Rothia, Veillonella, Erysipelatoclostridium, and Actinomyces compared with HCs. These results were consistent with our previous study (Zhou et al., 2021) on gut microbiota metagenomics of COVD-19 in the acute phase, in which we also found that the relative abundances of Streptococcus, Rothia, Veillonella, and Actinomyces were significantly higher in COVID-19 patients than in HCs, however, this study did not find these taxa differences at three months after discharge between survivors of COVID-19 and $\mathrm{HCs}$, indicating the relative abundance of these taxa gradually reduced or returned to normal at long term after discharge in survivors of COVID-19. All of these results suggest that the gut microbiota of COVID-19 dynamically changes.

Compared with HCs, the fecal microbiota of recovered HCWs at three months after discharge exhibited decreased bacterial diversity. Although only shannon index decreased significantly $(p=0.016)$, sobs index, chao index, and ace index also decreased. Low bacterial diversity has been reported in various diseases (Kuang et al., 2017; Yu et al., 2017; Wei et al., 2020), including COVID-19 in the acute phase (Gu et al., 2020), implying gut microbiota dysbiosis which may be related to the severity, course and prognosis of these diseases. We also observed an increase in opportunistic pathogens and inflammation-related pathogens, including Escherichia unclassified, I. bartlettii, C. aldenense, C. ramosum, C. bolteae, and F. plautii. Escherichia is composed of several species and cryptic clades, including Escherichia coli, as a vertebrate gut commensal, and occasionally as an opportunistic pathogen involved in diarrheic and extra-intestinal diseases (Galardini et al., 2020). Intestinibacter bartlettii was found to be more frequently present in patients with neurodevelopmental disorders patients group (Bojović et al., 2020), and previously known as Clostridium bartlettii isolated from the feces of two autistic male children (Song et al., 2004), is a strong producer of trans-3-Indolylacrylic acid (IAA), which can be transformed by glycine conjugation to Indolyl-3-acryloylglycine (IAG), a putative autistic spectrum disorder (ASD) diagnostic urinary marker (Shattock and Whiteley, 2002). Clostridium aldenense and C. bolteae are believed to cause bacteremia (Williams et al., 2010), and F. plautii has potential significance for the diagnosis of ulcerative colitis (UC) (Li et al., 2021). For the gut microbiota of COVID-19 in the acute phase, Zuo et al. (2020b) found that C. ramosum is correlated with COVID19 severity. In addition, previous studies have shown that F. prausnitzii (Lopez-Siles et al., 2017), R. inulinivorans (Ticinesi et al., 2020), F. saccharivorans (Jin et al., 2019), R. bromii (Rosés et al., 2021), B. faecis (Takahashi et al., 2016), B. pullicaecorum (Chang et al., 2020), and I. butyriciproducens (Bui et al., 2016) are all butyrate-producing species. Our research found that the above of butyrate-producing species were all reduced in recovered HCWs. Consistently, Yeoh et al. (2021) also found F. prausnitzii, R. bromii, and Dorea longicatena were reduced in COVID-19 patients and remained low in samples collected up to 30 days. Faecalibacterium prausnitzii is the most abundant bacterium in the human intestinal microbiota of healthy adults, representing more than $5 \%$ of the total bacterial population (Miquel et al., 2013; LopezSiles et al., 2017; Leylabadlo et al., 2020), which has anti-in- flammatory properties and produces short-chain fatty acids (SCFA) and stimulates the secretion of IL-10 through peripheral blood mononuclear cells, mucosal dendritic cells (DCs), and macrophages (Furet et al., 2010). SCFAs are end products of bacterial anaerobic fermentation of dietary fiber and can regulate immune responses in the intestines (MartinGallausiaux et al., 2021), such as by inducing regulatory $\mathrm{T}$ cells in the colon (Arpaia et al., 2013; Furusawa et al., 2013), inhibiting inflammatory responses through NF- $\kappa \mathrm{B}$ inhibition (Segain et al., 2000).

In this study, another significant finding was that altered gut microbiota was correlated with persistent symptoms after discharge. In recovered HCWs, Escherichia unclassified as an opportunistic pathogen was positively correlated with fatigue, chest tightness after activity, and myalgia. Intestinibacter bartlettii was positively correlated with anorexia and fatigue. Interestingly, F. prausnitzii and I. butyriciproducens as butyrate-producing species were negatively correlated with chest tightness after activity and cough, respectively. The above information suggests that gut microbiome dysbiosis may cause persistent symptoms of survivors of COVID-19 for a long time after discharge which may through regulating production of anti-inflammatory metabolites such as SCFA and/or facilitating the enrichment of opportunistic pathogens in the patients. However, the specific mechanism by which the gut microbiota affects recovered patients requires further study.

This study has several limitations. First, this was a singlecenter study with small sample size. However, regardless of recovered patients with COVID-19 or healthy controls were all healthcare workers from Wuhan Union Hospital, the collection process of stool samples was very strict. Although the sample size was small, the sample quality was very high. Second, our study only described the gut microbiota of recovered patients at three months after discharge. However, we do not know how long the altered gut microbiota will last. We should continue pay attention to gut microbiota at six months or even one year after discharge. Therefore, our team is currently collecting stool samples from recovered patients with COVID-19 at one year after discharge to assess whether the gut microbiota continues to be abnormal or return to normal. Third, lifestyle and diet may affect gut microbiota, we did not have information on lifestyle, however, the diet of all healthcare workers was provided by the worker restaurant of Wuhan Union Hospital.

In conclusion, this study provides evidence of gut microbiome dysbiosis in recovered HCWs with COVID-19 at three months after discharge and its association with persistent symptoms after discharge. These data highlight that gut microbiota may play an important role in the recovery of patients with COVID-19. The mechanism on how the gut microbiota alteration impacts the recovery of survivors of COVID-19 should be further studied.

\section{Acknowledgements}

This study gratefully acknowledges the patients who participated in the research. This work was supported by the Fundamental Research Funds for the Central Universities (No. 2020kfyXGYJ034) and (No. 2020kfyXGYJ009). 


\section{Conflict of Interest}

The authors declare that there is no conflict of interest.

\section{Ethical Statement}

The study was approved by the Ethics Committee of the Wuhan Union Hospital (2020-0149-02). All participants provided written informed consent prior to participation.

\section{Availability of Data and Materials}

The sequences from our study were deposited in the NCBI Sequence Read Archive (PRJNA736160).

\section{References}

Arpaia, N., Campbell, C., Fan, X., Dikiy, S., van der Veeken, J., deRoos, P., Liu, H., Cross, J.R., Pfeffer, K., Coffer, P.J., et al. 2013. Metabolites produced by commensal bacteria promote peripheral regulatory T-cell generation. Nature 504, 451-455.

Belkaid, Y. and Hand, T.W. 2014. Role of the microbiota in immunity and inflammation. Cell 157, 121-141.

Bojović, K., Ignjatović, Đ., Soković Bajić, S., Vojnović Milutinović, D., Tomić, M., Golić, N., and Tolinaćki, M. 2020. Gut microbiota dysbiosis associated with altered production of short chain fatty acids in children with neurodevelopmental disorders. Front. Cell. Infect. Microbiol. 10, 223.

Bui, T.P., Shetty, S.A., Lagkouvardos, I., Ritari, J., Chamlagain, B., Douillard, F.P., Paulin, L., Piironen, V., Clavel, T., Plugge, C.M., et al. 2016. Comparative genomics and physiology of the butyrateproducing bacterium Intestinimonas butyriciproducens. Environ. Microbiol. Rep. 8, 1024-1037.

Chan, J.F.W., Yuan, S., Kok, K.H., To, K.K.W., Chu, H., Yang, J., Xing, F., Liu, J., Yip, C.C.Y., Poon, R.W.S., et al. 2020. A familial cluster of pneumonia associated with the 2019 novel coronavirus indicating person-to-person transmission: a study of a family cluster. Lancet 395, 514-523.

Chang, S.C., Shen, M.H., Liu, C.Y., Pu, C.M., Hu, J.M., and Huang, C.J. 2020. A gut butyrate-producing bacterium Butyricicoccus pullicaecorum regulates short-chain fatty acid transporter and receptor to reduce the progression of 1,2-dimethylhydrazineassociated colorectal cancer. Oncol. Lett. 20, 327.

Furet, J.P., Kong, L.C., Tap, J., Poitou, C., Basdevant, A., Bouillot, J.L., Mariat, D., Corthier, G., Doré, J., Henegar, C., et al. 2010. Differential adaptation of human gut microbiota to bariatric surgeryinduced weight loss: links with metabolic and low-grade inflammation markers. Diabetes 59, 3049-3057.

Furusawa, Y., Obata, Y., Fukuda, S., Endo, T.A., Nakato, G., Takahashi, D., Nakanishi, Y., Uetake, C., Kato, K., Kato, T., et al. 2013. Commensal microbe-derived butyrate induces the differentiation of colonic regulatory T cells. Nature 504, 446-450.

Galardini, M., Clermont, O., Baron, A., Busby, B., Dion, S., Schubert, S., Beltrao, P., and Denamur, E. 2020. Major role of iron uptake systems in the intrinsic extra-intestinal virulence of the genus Escherichia revealed by a genome-wide association study. PLoS Genet. 16, e1009065.

Gu, S., Chen, Y., Wu, Z., Chen, Y., Gao, H., Lv, L., Guo, F., Zhang, X, Luo, R., Huang, C., et al. 2020. Alterations of the gut microbiota in patients with coronavirus disease 2019 or H1N1 influenza. Clin. Infect. Dis. 71, 2669-2678.
Huang, C., Wang, Y., Li, X., Ren, L., Zhao, J., Hu, Y., Zhang, L., Fan, G., Xu, J., Gu, X., et al. 2020. Clinical features of patients infected with 2019 novel coronavirus in Wuhan, China. Lancet 395, 497506.

Ichinohe, T., Pang, I.K., Kumamoto, Y., Peaper, D.R, Ho, J.H., Murray, T.S., and Iwasaki, A. 2011. Microbiota regulates immune defense against respiratory tract influenza A virus infection. Proc. Natl. Acad. Sci. USA 108, 5354-5359.

Jin, M., Kalainy, S., Baskota, N., Chiang, D., Deehan, E.C., McDougall, C., Tandon, P., Martínez, I., Cervera, C., Walter, J., et al. 2019. Faecal microbiota from patients with cirrhosis has a low capacity to ferment non-digestible carbohydrates into short-chain fatty acids. Liver Int. 39, 1437-1447.

Kuang, Y.S., Lu, J.H., Li, S.H., Li, J.H., Yuan, M.Y., He, J.R., Chen, N.N., Xiao, W.Q., Shen, S.Y., Qiu, L., et al. 2017. Connections between the human gut microbiome and gestational diabetes mellitus. GigaScience 6, 1-12.

Leylabadlo, H.E., Ghotaslou, R., Feizabadi, M.M., Farajnia, S., Moaddab, S.Y., Ganbarov, K., Khodadadi, E., Tanomand, A., Sheykhsaran, E., Yousefi, B., et al. 2020. The critical role of Faecalibacterium prausnitzii in human health: an overview. Microb. Pathog. 149, 104344.

Li, W., Sun, Y., Dai, L., Chen, H., Yi, B., Niu, J., Wang, L., Zhang, F., Luo, J., Wang, K., et al. 2021. Ecological and network analyses identify four microbial species with potential significance for the diagnosis/treatment of ulcerative colitis (UC). BMC Microbiol. 21, 138.

Liang, L., Yang, B., Jiang, N., Fu, W., He, X., Zhou, Y., Ma, W.L., and Wang, X. 2020. Three-month follow-up study of survivors of coronavirus disease 2019 after discharge. J. Korean Med. Sci. 35, e418.

Lopez-Siles, M., Duncan, S.H., Garcia-Gil, L.J., and Martinez-Medina, M. 2017. Faecalibacterium prausnitzii: from microbiology to diagnostics and prognostics. ISME J. 11, 841-852.

Martin-Gallausiaux, C., Marinelli, L., Blottière, H.M., Larraufie, P., and Lapaque, N. 2021. SCFA: mechanisms and functional importance in the gut. Proc. Nutr. Soc. 80, 37-49.

Miquel, S., Martín, R., Rossi, O., Bermúdez-Humarán, L.G., Chatel, J.M., Sokol, H., Thomas, M., Wells, J.M., and Langella, P. 2013. Faecalibacterium prausnitzii and human intestinal health. Curr. Opin. Microbiol. 16, 255-261.

Rosés, C., Cuevas-Sierra, A., Quintana, S., Riezu-Boj, J.I., Martínez, J.A., Milagro, F.I., and Barceló, A. 2021. Gut microbiota bacterial species associated with mediterranean diet-related food groups in a northern spanish population. Nutrients 13, 636.

Schirmer, M., Smeekens, S.P., Vlamakis, H., Jaeger, M., Oosting, M., Franzosa, E.A., Ter Horst, R., Jansen, T., Jacobs, L., Bonder, M.J., et al. 2016. Linking the human gut microbiome to inflammatory cytokine production capacity. Cell 167, 1125-1136.

Segain, J.P., de la Blétière, D.R., Bourreille, A., Leray, V., Gervois, N., Rosales, C., Ferrier, L., Bonnet, C., Blottière, H.M., and Galmiche, J.P. 2000. Butyrate inhibits inflammatory responses through NFkB inhibition: implications for Crohn's disease. Gut 47, 397403.

Shattock, P. and Whiteley, P. 2002. Biochemical aspects in autism spectrum disorders: updating the opioid-excess theory and presenting new opportunities for biomedical intervention. Expert Opin. Ther. Targets 6, 175-183.

Song, Y.L., Liu, C.X., McTeague, M., Summanen, P., and Finegold, S.M. 2004. Clostridium bartlettii sp. nov., isolated from human faeces. Anaerobe 10, 179-184.

Takahashi, K., Nishida, A., Fujimoto, T., Fujii, M., Shioya, M., Imaeda, H., Inatomi, O., Bamba, S., Sugimoto, M., and Andoh, A. 2016. Reduced abundance of butyrate-producing bacteria species in the fecal microbial community in Crohn's disease. Digestion 93, 59-65.

Ticinesi, A., Mancabelli, L., Tagliaferri, S., Nouvenne, A., Milani, C., 
Del Rio, D., Lauretani, F., Maggio, M.G., Ventura, M., and Meschi, T. 2020. The gut-muscle axis in older subjects with low muscle mass and performance: a proof of concept study exploring fecal microbiota composition and function with shotgun metagenomics sequencing. Int. J. Mol. Sci. 21, 8946.

Wang, X., Zhou, Q., He, Y., Liu, L., Ma, X., Wei, X., Jiang, N., Liang, L., Zheng, Y., Ma, L., et al. 2020a. Nosocomial outbreak of COVID19 pneumonia in Wuhan, China. Eur. Respir. J. 55, 2000544.

Wang, X., Zhou, Y., Jiang, N., Zhou, Q., and Ma, W.L. 2020b. Persistence of intestinal SARS-CoV-2 infection in patients with COVID19 leads to re-admission after pneumonia resolved. Int. J. Infect. Dis. 95, 433-435.

Wei, Y., Li, Y., Yan, L., Sun, C., Miao, Q., Wang, Q., Xiao, X., Lian, M., Li, B., Chen, Y., et al. 2020. Alterations of gut microbiome in autoimmune hepatitis. Gut 69, 569-577.

Williams, O.M., Brazier, J., Peraino, V., and Goldstein, E.J. 2010. A review of three cases of Clostridium aldenense bacteremia. Anaerobe 16, 475-477.

Yeoh, Y.K., Zuo, T., Lui, G.C.Y., Zhang, F., Liu, Q., Li, A.Y.L., Chung, A.C.K., Cheung, C.P., Tso, E.Y.K., Fung, K.S.C., et al. 2021. Gut microbiota composition reflects disease severity and dysfunctional immune responses in patients with COVID-19. Gut 70
698-706.

Yu, J., Feng, Q., Wong, S.H., Zhang, D., Liang, Q.Y., Qin, Y., Tang, L., Zhao, H., Stenvang, J., Li, Y., et al. 2017. Metagenomic analysis of faecal microbiome as a tool towards targeted non-invasive biomarkers for colorectal cancer. Gut 66, 70-78.

Zhou, Y., Shi, X., Fu, W., Xiang, F., He, X., Yang, B., Wang, X., and Ma, W.L. 2021. Gut microbiota dysbiosis correlates with abnormal immune response in moderate COVID-19 patients with fever. $J$. Inflamm. Res. 14, 2619-2631.

Zuo, T., Liu, Q., Zhang, F., Lui, G.C., Tso, E.Y., Yeoh, Y.K., Chen, Z., Boon, S.S., Chan, F.K., Chan, P.K., et al. 2021. Depicting SARSCoV-2 faecal viral activity in association with gut microbiota composition in patients with COVID-19. Gut 70, 276-284.

Zuo, T., Zhan, H., Zhang, F., Liu, Q., Tso, E.Y.K., Lui, G.C.Y., Chen, N., Li, A., Lu, W., Chan, F.K.L., et al. 2020a. Alterations in fecal fungal microbiome of patients with COVID-19 during time of hospitalization until discharge. Gastroenterology 159, 1302-1310.

Zuo, T., Zhang, F., Lui, G.C.Y., Yeoh, Y.K., Li, A.Y.L., Zhan, H., Wan, Y., Chung, A.C.K., Cheung, C.P., Chen, N., et al. 2020b. Alterations in gut microbiota of patients with COVID-19 during time of hospitalization. Gastroenterology 159, 944-955. 